\title{
QUASILINEAR NONHOMOGENEOUS SCHRÖDINGER EQUATION WITH CRITICAL EXPONENTIAL GROWTH IN $\mathbb{R}^{n}$
}

\author{
Manassés de Souza - João Marcos do Ó - Tarciana Silva
}

\begin{abstract}
In this paper, using variational methods, we establish the existence and multiplicity of weak solutions for nonhomogeneous quasilinear elliptic equations of the form

$$
-\Delta_{n} u+a(x)|u|^{n-2} u=b(x)|u|^{n-2} u+g(x) f(u)+\varepsilon h \text { in } \mathbb{R}^{n},
$$

where $n \geq 2, \Delta_{n} u \equiv \operatorname{div}\left(|\nabla u|^{n-2} \nabla u\right)$ is the $n$-Laplacian and $\varepsilon$ is a positive parameter. Here the function $g(x)$ may be unbounded in $x$ and the nonlinearity $f(s)$ has critical growth in the sense of Trudinger-Moser inequality, more precisely $f(s)$ behaves like $e^{\alpha_{0}|s|^{n /(n-1)}}$ when $s \rightarrow+\infty$ for some $\alpha_{0}>0$. Under some suitable assumptions and based on a TrudingerMoser type inequality, our results are proved by using Ekeland variational principle, minimization and mountain-pass theorem.
\end{abstract}

\section{Introduction}

This paper is concerned with the existence and multiplicity of solutions for nonhomogeneous quasilinear elliptic equations of the form

$$
-\Delta_{n} u+a(x)|u|^{n-2} u=b(x)|u|^{n-2} u+g(x) f(u)+\varepsilon h \quad \text { in } \mathbb{R}^{n},
$$

2010 Mathematics Subject Classification. 35J20, 35J60, 35B33.

Key words and phrases. Variational methods, Trudinger-Moser inequality, critical points, critical exponents, $n$-Laplacian.

Research partially supported by the National Institute of Science and Technology of Mathematics INCT-Mat, CAPES and CNPq. 
where $n \geq 2, \Delta_{n} u \equiv \operatorname{div}\left(|\nabla u|^{n-2} \nabla u\right)$ is the $n$-Laplacian, $\varepsilon$ is a positive parameter, $a, b, g: \mathbb{R}^{n} \rightarrow[0,+\infty)$ and $f: \mathbb{R} \rightarrow \mathbb{R}$ are functions satisfying mild conditions and $h$ belongs to the dual of an appropriated subspace $E$ of the Sobolev space $W^{1, n}\left(\mathbb{R}^{n}\right)$. Here, we are interested when $f(s)$ has the maximal growth which allows to treat equation (1.1) variationally in $E$, in the sense that $\int g(x) F(u) d x<\infty$ if $u \in E$, where $F(s)=\int_{0}^{s} f(t) d t$. In the so-called Sobolev case $1<p<n$, the Sobolev embedding asserts that $W^{1, p}\left(\mathbb{R}^{n}\right) \hookrightarrow L^{r}\left(\mathbb{R}^{n}\right)$ for any $1<r \leq p^{*}$, where $p^{*}=n p /(n-p)$ is the critical Sobolev exponent. Consequently, for this case, the maximal growth is naturally given by the Sobolev exponent (cf. [26]). In the borderline case $p=n$, formally $p^{*} \rightsquigarrow \infty$, but $W^{1, n}\left(\mathbb{R}^{n}\right) \hookrightarrow L^{\infty}\left(\mathbb{R}^{n}\right)$. For this case, the maximal growth is motivated by an inequality of Trudinger-Moser type which ensures that $W^{1, n}\left(\mathbb{R}^{n}\right)$ is embedded in an Orlicz space generated by the Young function $\phi(s) \sim e^{\alpha_{n}|s|^{n /(n-1)}}$ as $|s| \rightarrow \infty$, where $\alpha_{n}:=n \omega_{n-1}^{1 /(n-1)}$ and $\omega_{n-1}$ is the measure of the unit sphere in $\mathbb{R}^{n}$ (cf. [1], [7], [8], [13], [21], [22], [25]).

Since (1.1) is of variational type, we can see that solutions of (1.1) correspond to critical point of the associated energy functional and to obtain the existence and multiplicity of solutions of (1.1) we will apply minimax methods, more precisely, the Ekeland variational principle combined with minimization and the mountain-pass theorem. For that we will assume suitable conditions on the potential $a(x)$ with which we will be able to consider a variational framework based in the subspace $E$ of $W^{1, n}\left(\mathbb{R}^{n}\right)$ given by

$$
E=\left\{u \in W^{1, n}\left(\mathbb{R}^{n}\right): \int_{\mathbb{R}^{n}} a(x)|u|^{n} d x<\infty\right\}
$$

which is a Banach space when endowed with the norm

$$
\|u\|=\left(\int_{\mathbb{R}^{n}}\left(|\nabla u|^{n}+a(x)|u|^{n}\right) d x\right)^{1 / n} .
$$

Moreover, using the Clarkson's first inequality (see [4, p. 95]) it follows that $E$ is uniformly convex, and thus reflexive.

Fixed $h$ in the dual space $E^{\prime}$ of $E$, we will look for $u \in E$ weak solution of problem (1.1) in the following sense

$\int_{\mathbb{R}^{n}}\left[\left(|\nabla u|^{n-2} \nabla u \nabla v+a(x)|u|^{n-2} u v\right)-b(x)|u|^{n-2} u v-g(x) f(u) v\right] d x-\varepsilon\langle h, v\rangle=0$,

for all $v \in E$, where $\langle\cdot, \cdot\rangle$ denotes the duality pairing between $E$ and its dual space $E^{\prime}$ with associated norm denoted by $\|\cdot\|_{E^{\prime}}$.

In order to state our main results, let us introduce the assumptions that we assume throughout this article:

$\left(\mathrm{a}_{1}\right)$ The function $a: \mathbb{R}^{n} \rightarrow[0,+\infty)$ is measurable and $a \in L_{\mathrm{loc}}^{\infty}\left(\mathbb{R}^{n}\right)$. 
$\left(\mathrm{a}_{2}\right)$ The infimum $\lambda_{1}:=\inf \left\{\int_{\mathbb{R}^{n}}\left(|\nabla u|^{n}+a(x)|u|^{n}\right) d x: u \in E\right.$ and $\left.\|u\|_{n}=1\right\}$ is positive.

Consider the space $E$ endowed with the norm given in (1.2). Suppose that the conditions $\left(a_{1}\right)-\left(a_{2}\right)$ are valid. It is easy to see that the following embedding are continuous:

$$
E \hookrightarrow W^{1, n}\left(\mathbb{R}^{n}\right) \hookrightarrow L^{s}\left(\mathbb{R}^{n}\right) \quad \text { for all } s \in[n,+\infty) .
$$

We use the following notation: if $\Omega \subset \mathbb{R}^{n}$ is open and $s \in[n,+\infty)$, we set

$$
\nu_{s}(\Omega)= \begin{cases}\inf _{u \in W_{0}^{1, n}(\Omega) \backslash\{0\}} \frac{\int_{\Omega}\left(|\nabla u|^{n}+a(x)|u|^{n}\right) d x}{\left(\int_{\Omega}|u|^{s} d x\right)^{n / s}} & \text { if } \Omega \neq \emptyset, \\ \infty & \text { if } \Omega=\emptyset .\end{cases}
$$

In order to obtain a compactness result, we shall consider the following assumptions:

(a) $\lim _{R \rightarrow \infty} \nu_{n}\left(\mathbb{R}^{n} \backslash \bar{B}_{R}\right)=\infty$;

$\left(\mathrm{a}_{4}\right)$ There exist a function $A(x) \in L_{\text {loc }}^{\infty}\left(\mathbb{R}^{n}\right)$, with $A(x) \geq 1$, and constants $\beta>1, c_{0}, R_{0}>0$ such that

$$
A(x) \leq c_{0}\left[1+(a(x))^{1 / \beta}\right], \quad \text { for all }|x| \geq R_{0} .
$$

We will prove in Lemma 2.4 that under assumptions $\left(\mathrm{a}_{3}\right)-\left(\mathrm{a}_{4}\right)$, the space $E$ is compactly embedding into $L^{s}\left(\mathbb{R}^{n}\right)$ for all $s \in[n,+\infty)$.

Concerning the function $g(x)$, we assume that it is strictly positive and does not have to be bounded in $x$ provided that the growth of $g(x)$ is controlled by the growth of $a(x)$. More precisely,

$\left(\mathrm{g}_{1}\right) g: \mathbb{R}^{n} \rightarrow[0,+\infty)$ is continuous and there exist $\lambda_{0}, \Lambda_{0}>0$ such that

$$
\lambda_{0} \leq g(x) \leq \Lambda_{0} A(x), \quad \text { for all } x \in \mathbb{R}^{n} .
$$

We assume the following assumptions on the nonlinear term:

$\left(\mathrm{f}_{1}\right) f: \mathbb{R} \rightarrow \mathbb{R}$ is continuous and $\lim _{s \rightarrow 0} f(s) s^{1-n}=0$.

$\left(\mathrm{f}_{2}\right) f$ has critical growth, that is, there exists $\alpha_{0}>0$ such that

$$
\lim _{|s| \rightarrow+\infty} f(s) e^{-\alpha|s|^{n /(n-1)}}= \begin{cases}0 & \text { for all } \alpha>\alpha_{0}, \\ +\infty & \text { for all } \alpha<\alpha_{0} .\end{cases}
$$

$\left(\mathrm{f}_{3}\right)$ There is $\mu>n$ such that

$$
0<\mu F(s) \leq s f(s), \quad \text { for all } s \in \mathbb{R} \backslash\{0\} .
$$

$\left(\mathrm{f}_{4}\right)$ There exist constants $S_{0}, M_{0}>0$ such that

$$
0<F(s) \leq M_{0}|f(s)|, \quad \text { for all }|s| \geq S_{0} .
$$


Concerning the function $b(x)$, we assume that

$\left(\mathrm{b}_{1}\right) b: \mathbb{R}^{n} \rightarrow[0,+\infty)$ is a measurable function such that $\|b\|_{\sigma}<S_{t_{0}}^{n}$, where $t_{0}:=\sigma n /(\sigma-1)$ for some $\sigma>1$ and $S_{t_{0}}$ is the best constant for the Sobolev embedding $E \hookrightarrow L^{t_{0}}\left(\mathbb{R}^{n}\right)$, that is,

$$
S_{t_{0}}:=\inf _{u \in E \backslash\{0\}} \frac{\left(\int_{\mathbb{R}^{n}}\left(|\nabla u|^{n}+a(x)|u|^{n}\right) d x\right)^{1 / n}}{\left(\int_{\mathbb{R}^{n}}|u|^{t_{0}} d x\right)^{1 / t_{0}}} .
$$

Next we state our main results.

Theorem 1.1. Suppose that $\left(\mathrm{a}_{1}\right)-\left(\mathrm{a}_{4}\right),\left(\mathrm{g}_{1}\right),\left(\mathrm{f}_{1}\right)-\left(\mathrm{f}_{3}\right)$ and $\left(\mathrm{b}_{1}\right)$ are satisfied. Then there exists $\varepsilon_{1}>0$ such that for each $\varepsilon \in\left(0, \varepsilon_{1}\right)$, problem (1.1) possesses a weak solution with negative energy.

Theorem 1.2. Suppose that $\left(\mathrm{a}_{1}\right)-\left(\mathrm{a}_{4}\right),\left(\mathrm{g}_{1}\right),\left(\mathrm{f}_{1}\right)-\left(\mathrm{f}_{4}\right)$ and $\left(\mathrm{b}_{1}\right)$ are satisfied. Furthermore suppose that

$\left(\mathrm{f}_{5}\right)$ there exists $p>n$ such that

$$
f(s) \geq C_{p} s^{p-1}, \quad \text { for all } s \geq 0,
$$

where

$$
C_{p}>\left[\frac{S_{p}^{p}}{\lambda_{0}}\left(\frac{\beta n}{\beta-1}\right)^{p-n}\left(\frac{p-n}{p}\right)^{(p-n) / n}\left(\frac{\alpha_{0}}{\alpha_{n}}\right)^{(n-1)(p-n) / n}\right] .
$$

Then there exists $\varepsilon_{2}>0$ such that for each $\varepsilon \in\left(0, \varepsilon_{2}\right)$, problem (1.1) possesses a second weak solution.

TheOREM 1.3. Suppose that $h \equiv 0$ and $\left(\mathrm{a}_{1}\right)-\left(\mathrm{a}_{4}\right),\left(\mathrm{g}_{1}\right),\left(\mathrm{f}_{1}\right)-\left(\mathrm{f}_{5}\right),\left(\mathrm{b}_{1}\right)$ are satisfied. Then problem (1.1) possesses a nontrivial weak solution.

REMARK 1.4. We point out that the existence of solutions for the quasilinear elliptic problem

$$
-\Delta_{n} u+a(x)|u|^{n-2} u=p(x, u), \quad x \in \mathbb{R}^{n},
$$

with $n \geq 2$ has been discussed recently under various conditions on the potential $a(x)$ and the nonlinearity $p(x, s)$. For more details on this subject, we refer the reader to the papers [2], [9], [11], [13], [14], [16], [17], [19], [27] and references therein. It is worthwhile to remark that in these works different hypotheses are assumed on $a(x)$ in order to overcome the problem of "lack of compactness", typical of elliptic problems defined in unbounded domains and involving nonlinearities in critical growth range. More precisely, in many papers it is usually assumed that the potential is continuous and uniformly positive, that is, $a(x) \geq a_{0}>0$ for any $x \in \mathbb{R}^{n}$. Furthermore it is assumed one of the following conditions: 
(a) $a(x) \rightarrow+\infty$ as $|x| \rightarrow+\infty$;

(b) $[a(x)]^{-1} \in L^{1}\left(\mathbb{R}^{n}\right)$;

(c) For any $L>0$, the level set $\left\{x \in \mathbb{R}^{n}: a(x) \leq L\right\}$ has finite Lebesgue measure.

Each of these conditions guarantee that the space $E:=\left\{u \in W^{1, n}\left(\mathbb{R}^{n}\right)\right.$ : $\left.\int_{\mathbb{R}^{n}} a(x)|u|^{n} d x<\infty\right\}$ is compactly embedded in the Lebesgue space $L^{s}\left(\mathbb{R}^{n}\right)$ for all $s \geq n$. Moreover, it is assumed that there exists $\alpha_{0}>0$ such that

$$
|p(x, s)| \leq c_{1}|s|^{n-1}+c_{2} \Phi_{\alpha_{0}}(s), \quad \text { for all }(x, s) \in \mathbb{R}^{n} \times \mathbb{R},
$$

which implies that $p(x, s)$ is bounded with respect to the variable $x$.

REMARK 1.5. Note that a sufficient condition for the hypothesis $\left(\mathrm{a}_{3}\right)$ is that

$$
\lim _{R \rightarrow \infty} \mathcal{L}\left(\Omega_{L} \cap\left(\mathbb{R}^{n} \backslash \bar{B}_{R}\right)\right)=0, \quad \text { for all } L>0,
$$

where $\Omega_{L}=\left\{x \in \mathbb{R}^{n}: a(x)<L\right\}$ and $\mathcal{L}(\Omega)$ is the Lebesgue measure of $\Omega$. Thus, the potentials satisfying (a)-(c) also satisfy the condition $\left(\mathrm{a}_{3}\right)$. Consequently, the condition $\left(a_{3}\right)$ improves $(a)-(c)$. Should be stressed that our approach covers the case when the potential $a(x)$ may vanish and the nonlinear term admits general growth and can be unbounded in variable $x$.

REMARK 1.6. In the semilinear case which corresponds to

$$
-\Delta u+a(x) u=p(x, u) \quad \text { in } \mathbb{R}^{n}
$$

such class of equations arise in various branches of mathematical physics and they have been the subject of extensive study in recent years. Part of the interest is due to the fact that solutions of (1.4) are related to the existence of solitary wave solutions for nonlinear Schrödinger equations and Klein-Gordon equations (for a discussion see for example [3]). Our work was motivated by some papers that have appeared in the recent years concerning the study of (1.4) by using purely variational approach since the seminal work of Rabinowitz [23]. In special should be mentioned that the condition $\left(\mathrm{a}_{3}\right)$ was already considered by B. Sirakov [24] to study (1.4) when $n \geq 3$ and $p(x, u)$ is superlinear and has subcritical growth in the Sobolev sense. Our main purpose is to extend and complement the results in [24] to consider critical growth in the Trudinger-Moser sense. Similar to B. Sirakov [24, Proposition 3.1] we also prove here a compactness result for the borderline case which is another important point of the present paper (cf. Lemma 2.4).

The remaining part of this paper is organized as follows: In Section 2, we have some technical results, and in particular, we prove the crucial fact that the space $E$ is compactly embedded in some Lebesgue spaces. In Section 3, we introduce the variational framework and we study some geometric properties of the functional $I$. In Section 4 we obtain an estimate to the mountain pass level 
of $I$. In Section 5 we analyze the Palais-Smale compactness of functional $I$. Theorems 1.1 and 1.2 are proved in Section 6. Finally Theorem 1.3 is proved in Section 7 .

\section{Some preliminary results}

In this section, we obtain some technical results which will be used in the proof of main results. In [5] for $n=2$ and [13] for $n \geq 2$ was proved the following version of the Trudinger-Moser inequality to the whole $\mathbb{R}^{n}$ :

Lemma 2.1. If $\alpha>0$ and $u \in W^{1 . n}\left(\mathbb{R}^{n}\right)$ then

$$
\int_{\mathbb{R}^{n}} \Phi_{\alpha}(u) d x<\infty
$$

where

$$
\Phi_{\alpha}(s):=e^{\alpha|s|^{n /(n-1)}}-\sum_{j=0}^{n-2} \frac{\alpha^{j}|s|^{j n /(n-1)}}{j !} .
$$

Moreover, if $0<\alpha<\alpha_{n},\|\nabla u\|_{n} \leq 1$ and $\|u\|_{n} \leq T$, then there is a positive constant $C=C(\alpha, T)$ such that

$$
\int_{\mathbb{R}^{n}} \Phi_{\alpha}(u) d x \leq C(\alpha, T),
$$

where $\alpha_{n}:=n \omega_{n-1}^{1 /(n-1)}$ and $\omega_{n-1}$ is the measure of the unit sphere in $\mathbb{R}^{n}$.

Let us consider the weighted Lebesgue space $L_{A(x)}^{s}\left(\mathbb{R}^{n}\right)$ with the usual norm

$$
\|u\|_{L_{A(x)}^{s}\left(\mathbb{R}^{n}\right)}=\left(\int_{\mathbb{R}^{n}} A(x)|u|^{s} d x\right)^{1 / s} .
$$

Lemma 2.2. Assume that $\left(\mathrm{a}_{1}\right)-\left(\mathrm{a}_{2}\right)$ and $\left(\mathrm{a}_{4}\right)$ hold. Then $E$ is continuously embedding into $L_{A(x)}^{s}\left(\mathbb{R}^{n}\right)$ for $s \in[n,+\infty)$.

Proof. By using $\left(a_{4}\right)$ we get

$$
\begin{aligned}
& \int_{\mathbb{R}^{n}} A(x)|u|^{s} d x=\int_{|x|>R_{0}} A(x)|u|^{s} d x+\int_{|x| \leq R_{0}} A(x)|u|^{s} d x \\
& \quad \leq c_{0} \int_{|x|>R_{0}}\left[1+(a(x))^{1 / \beta}\right]|u|^{s} d x+\|A\|_{L^{\infty}\left(B_{R_{0}}\right)} \int_{|x| \leq R_{0}}|u|^{s} d x \\
& =c_{0} \int_{|x|>R_{0}}(a(x))^{1 / \beta}|u|^{s} d x \\
& \quad+c_{0} \int_{|x|>R_{0}}|u|^{s} d x+\|A\|_{L^{\infty}\left(B_{R_{0}}\right)} \int_{|x| \leq R_{0}}|u|^{s} d x \\
& \leq c_{0} \int_{|x|>R_{0}}(a(x))^{1 / \beta}|u|^{s} d x+\left(c_{0}+\|A\|_{L^{\infty}\left(B_{R_{0}}\right)}\right) \int_{\mathbb{R}^{n}}|u|^{s} d x \\
& \leq c_{0} \int_{|x|>R_{0}}(a(x))^{1 / \beta}|u|^{s} d x+\left(c_{0}+\|A\|_{L^{\infty}\left(B_{R_{0}}\right)}\right)\|u\|_{s}^{s} .
\end{aligned}
$$


Using Hölder's inequality, we obtain

$$
\begin{aligned}
\int_{|x|>R_{0}} & (a(x))^{1 / \beta}|u|^{s} d x=\int_{|x|>R_{0}}\left(a(x)|u|^{n}\right)^{1 / \beta}|u|^{s-n / \beta} d x \\
\leq & \left(\int_{|x|>R_{0}} a(x)|u|^{n} d x\right)^{1 / \beta}\left(\int_{|x|>R_{0}}\left(|u|^{s-n / \beta}\right)^{\beta /(\beta-1)} d x\right)^{(\beta-1) / \beta} .
\end{aligned}
$$

Consequently,

$$
\int_{|x|>R_{0}}(a(x))^{1 / \beta}|u|^{s} d x \leq\|u\|^{n / \beta}\|u\|_{(s \beta-n) /(\beta-1)}^{(s \beta-n) / \beta} .
$$

From (2.3) and (2.4), we get

$$
\int_{\mathbb{R}^{n}} A(x)|u|^{s} d x \leq C_{1}\|u\|_{s}^{s}+C_{2}\|u\|^{n / \beta}\|u\|_{(s \beta-n) /(\beta-1)}^{(s \beta-n) / \beta} .
$$

Since $(s-n / \beta)(\beta /(\beta-1)) \geq n$, using (1.3), we conclude that

$$
\|u\|_{L_{A(x)}^{s}}^{s}\left(\mathbb{R}^{n}\right) \leq C_{1}\|u\|^{s}+C_{2}\|u\|^{n / \beta}\|u\|^{(s \beta-n) / \beta} \leq C\|u\|^{s} .
$$

This complete the proof of Lemma 2.2.

To obtain our compactness result we need the following lemma.

Lemma 2.3. Let $\Omega \subset \mathbb{R}^{n}$ be an open subset. For each $s \in[n,+\infty)$ there exist $\theta_{1} \in(0,1)$ and $C>0$ such that

$$
\nu_{s}(\Omega) \geq C\left(\nu_{n}(\Omega)\right)^{\theta_{1}} .
$$

Proof. Using the Gagliardo-Nirenberg inequality [18, Proposition 8.12] we reach

$$
\|u\|_{s}^{n} \leq C\|u\|_{n}^{(1-\theta) n}\|\nabla u\|_{n}^{\theta n}, \quad \text { for all } u \in W_{0}^{1, n}(\Omega),
$$

where $1 / s=(1-\theta) / n$ and $\theta \in(0,1)$. Thus, by Lemma 2.2 there exists $C>0$ such that

$$
\|u\|_{s}^{n} \leq C\|u\|_{n}^{(1-\theta) n}\|u\|^{\theta n}, \quad \text { for all } u \in W_{0}^{1, n}(\Omega) .
$$

This together with the definition of $\nu_{s}(\Omega)$ implies

$$
\begin{aligned}
\nu_{s}(\Omega) & =\inf _{u \in W_{0}^{1, n}(\Omega) \backslash\{0\}} \frac{\int_{\mathbb{R}^{n}}|\nabla u|^{n}+a(x)|u|^{n}}{\|u\|_{s}^{n}} \geq \inf _{u \in W_{0}^{1, n}(\Omega) \backslash\{0\}} \frac{\|u\|^{n}}{C\|u\|_{n}^{(1-\theta) n}\|u\|^{\theta n}} \\
& =\frac{1}{C} \inf _{u \in W_{0}^{1, n}(\Omega) \backslash\{0\}} \frac{\|u\|^{n}}{\|u\|_{n}^{(1-\theta) n}\|u\|^{\theta n}}=\frac{1}{C} \inf _{u \in W_{0}^{1, n}(\Omega) \backslash\{0\}} \frac{\|u\|^{(1-\theta) n}}{\|u\|_{n}^{(1-\theta) n}} \\
& =\frac{1}{C} \inf _{u \in W_{0}^{1, n}(\Omega) \backslash\{0\}}\left(\frac{\|u\|^{n}}{\|u\|_{n}^{n}}\right)^{(1-\theta)}=\frac{1}{C}\left(\nu_{n}(\Omega)\right)^{(1-\theta)} .
\end{aligned}
$$

Therefore, (2.6) holds and the lemma is proved.

Lemma 2.4. Suppose that $\left(\mathrm{a}_{1}\right)-\left(\mathrm{a}_{4}\right)$ hold. Then $E$ is compactly embedded into $L^{s}\left(\mathbb{R}^{n}\right)$ and $L_{A(x)}^{s}\left(\mathbb{R}^{n}\right)$ for $s \in[n,+\infty)$. 
Proof. Take $\left(u_{k}\right) \subset E$ a bounded sequence. Thus, up to a subsequence, $u_{k} \rightarrow u$ weakly in $E$.

Assertion 1. Up to a subsequence, $u_{k} \rightarrow u$ strongly in $L^{s}\left(\mathbb{R}^{n}\right)$ for $s \in$ $[n,+\infty)$.

Indeed, let $\varphi \in C^{\infty}\left(\mathbb{R}^{n},[0,1]\right)$ be a function such that $\|\nabla \varphi\|_{\infty} \leq 1$ and

$$
\varphi(x)= \begin{cases}0 & \text { if }|x| \leq R \\ 1 & \text { if }|x|>R+1\end{cases}
$$

Thus,

$$
\begin{aligned}
\left\|u_{k}-u\right\|_{s} & =\left\|(1-\varphi)\left(u_{k}-u\right)+\varphi\left(u_{k}-u\right)\right\|_{s} \\
& \leq\left\|(1-\varphi)\left(u_{k}-u\right)\right\|_{s}+\left\|\varphi\left(u_{k}-u\right)\right\|_{s} \\
& =\left\|(1-\varphi)\left(u_{k}-u\right)\right\|_{L^{s}\left(B_{R+1}\right)}+\left\|\varphi\left(u_{k}-u\right)\right\|_{L^{s}\left(\mathbb{R}^{n} \backslash B_{R}\right)} .
\end{aligned}
$$

Since $W^{1, n}\left(B_{R+1}\right)$ is compactly embedded into $L^{s}\left(B_{R+1}\right)$ for $s \in[n, \infty)$, up to a subsequence, we get

$$
\left\|(1-\varphi)\left(u_{k}-u\right)\right\|_{L^{s}\left(B_{R+1}\right)} \rightarrow 0 .
$$

Now, by the definition of $\nu_{s}\left(\mathbb{R}^{n} \backslash B_{R}\right)$, it follows that

$$
\left\|\varphi\left(u_{k}-u\right)\right\|_{L^{s}\left(\mathbb{R}^{n} \backslash B_{R}\right)} \leq \frac{\int_{\mathbb{R}^{n} \backslash B_{R}}\left(\left|\nabla\left(\varphi\left(u_{k}-u\right)\right)\right|^{n}+a(x)\left|\varphi\left(u_{k}-u\right)\right|^{n}\right) d x}{\nu_{s}\left(\mathbb{R}^{n} \backslash B_{R}\right)} .
$$

Note that

$$
\begin{aligned}
\left\|\varphi\left(u_{k}-u\right)\right\|^{n} & =\int_{\mathbb{R}^{n}}\left|(\nabla \varphi)\left(u_{k}-u\right)\right|^{n}+\left|\varphi\left(\nabla\left(u_{k}-u\right)\right)\right|^{n}+a(x)\left|\varphi\left(u_{k}-u\right)\right|^{n} d x \\
& \leq \int_{\mathbb{R}^{n}}\left|u_{k}-u\right|^{n} d x+\int_{\mathbb{R}^{n}}\left|\nabla\left(u_{k}-u\right)\right|^{n}+a(x)\left|u_{k}-u\right|^{n} d x \\
& \leq\left\|u_{k}-u\right\|_{1, n}^{n}+\left\|u_{k}-u\right\|^{n} .
\end{aligned}
$$

Consequently, using the continuous embedding $E \hookrightarrow W^{1, n}\left(\mathbb{R}^{n}\right)$, we get

$$
\left\|\varphi\left(u_{k}-u\right)\right\|^{n} \leq C
$$

which together with (2.9), $\left(\mathrm{a}_{3}\right)$ and (2.6), implies

$$
\left\|\varphi\left(u_{k}-u\right)\right\|_{L^{s}\left(\mathbb{R}^{n} \backslash B_{R}\right)} \rightarrow 0 .
$$

From (2.7), (2.8) and (2.10) we conclude Assertion 1.

Assertion 2. Up to a subsequence, $u_{k} \rightarrow u$ strongly in $L_{A(x)}^{s}\left(\mathbb{R}^{n}\right)$ for $s \in$ $[n,+\infty)$.

From (2.5), we have

$$
\int_{\mathbb{R}^{n}} A(x)\left|u_{k}-u\right|^{s} d x \leq C_{1}\left\|u_{k}-u\right\|_{s}^{s}+C_{2}\left\|u_{k}-u\right\|^{n / \beta}\left\|u_{k}-u\right\|_{(s \beta-n) /(\beta-1)}^{(s \beta-n) / \beta} .
$$


Thus, as a consequence of Assertion 1 we get

$$
\int_{\mathbb{R}^{n}} A(x)\left|u_{k}-u\right|^{s} d x \rightarrow 0
$$

where we have used that $(s-n / \beta)(\beta /(\beta-1)) \geq n$. This completes the proof of Lemma 2.4.

Lemma 2.5. Suppose that $\left(\mathrm{a}_{1}\right)-\left(\mathrm{a}_{2}\right),\left(\mathrm{a}_{4}\right),\left(\mathrm{g}_{1}\right)$ and $\left(\mathrm{f}_{1}\right)-\left(\mathrm{f}_{2}\right)$ are satisfied. Then

$$
\int_{\mathbb{R}^{n}} A(x)|u|^{q} \Phi_{\alpha}(u) d x<\infty, \quad \text { for all } u \in E, q \geq n .
$$

Moreover, if $\alpha(\beta /(\beta-1))^{n /(n-1)}\|u\|^{n /(n-1)}<\alpha_{n}$, there exists $C>0$ such that

$$
\int_{\mathbb{R}^{n}} A(x)|u|^{q} \Phi_{\alpha}(u) d x \leq C\|u\|^{q}, \quad \text { for all } q \geq n .
$$

Proof. By using $\left(a_{4}\right)$ we get

$$
\begin{aligned}
& \int_{\mathbb{R}^{n}} A(x)|u|^{q} \Phi_{\alpha}(u) d x=\int_{|x|>R_{0}} A(x)|u|^{q} \Phi_{\alpha}(u) d x \\
&+\int_{|x| \leq R_{0}} A(x)|u|^{q} \Phi_{\alpha}(u) d x \\
& \leq c_{0} \int_{|x|>R_{0}}\left[1+(a(x))^{1 / \beta}\right]|u|^{q} \Phi_{\alpha}(u) d x \\
&+\|A\|_{L^{\infty}\left(B_{R_{0}}\right)} \int_{|x| \leq R_{0}}|u|^{q} \Phi_{\alpha}(u) d x \\
& \leq c_{0} \int_{|x|>R_{0}}(a(x))^{1 / \beta}|u|^{q} \Phi_{\alpha}(u) d x+c_{1} \int_{\mathbb{R}^{n}}|u|^{q} \Phi_{\alpha}(u) d x .
\end{aligned}
$$

Taking $s>1$ and $s^{\prime}$ such that $1 / s+1 / s^{\prime}=1$ and $1<s^{\prime}<\beta /(\beta-1)$, by Hölder's inequality and Lemma 2.1 in [27], we obtain

$\int_{\mathbb{R}^{n}}|u|^{q} \Phi_{\alpha}(u) d x \leq\|u\|_{q s}^{q}\left(\int_{\mathbb{R}^{n}}\left(\Phi_{\alpha}(u)\right)^{s^{\prime}} d x\right)^{1 / s^{\prime}} \leq\|u\|_{q s}^{q}\left(\int_{\mathbb{R}^{n}} \Phi_{\alpha}\left(s^{\prime} u\right) d x\right)^{1 / s^{\prime}}$.

Since $E \hookrightarrow L^{q s}\left(\mathbb{R}^{n}\right)$, using $(2.1)$ we get

$$
\int_{\mathbb{R}^{n}}|u|^{q} \Phi_{\alpha}(u) d x \leq C\|u\|^{q}\left(\int_{\mathbb{R}^{n}} \Phi_{\alpha}\left(s^{\prime} u\right) d x\right)^{1 / s^{\prime}}<\infty .
$$

Now, for each $u \in E$ with $\alpha(\beta /(\beta-1))^{n /(n-1)}\|u\|^{n /(n-1)}<\alpha_{n}$, it follows by (2.2) that there exists $C>0$ such that

$$
\int_{\mathbb{R}^{n}} \Phi_{\alpha}\left(s^{\prime} u\right) d x \leq C .
$$

From (2.12), we obtain that

$$
\int_{\mathbb{R}^{n}}|u|^{q} \Phi_{\alpha}(u) d x \leq C\|u\|^{q} .
$$


Let $\tau>1$ be sufficiently large and $\gamma>1$ such that $1 / \beta+1 / \tau+1 / \gamma=1$. By Hölder's inequality, we have

$$
\begin{aligned}
& \int_{|x|>R_{0}}(a(x))^{1 / \beta}|u|^{q} \Phi_{\alpha}(u) d x \leq\left(\int_{|x|>R_{0}} a(x)|u|^{n} d x\right)^{1 / \beta} \\
& \cdot\left(\int_{|x|>R_{0}}|u|^{(q-n / \beta) \tau} d x\right)^{1 / \tau}\left(\int_{|x|>R_{0}}\left(\Phi_{\alpha}(u)\right)^{\gamma} d x\right)^{1 / \gamma} .
\end{aligned}
$$

Again using Lemma 2.1 in [27], we have

$$
\begin{aligned}
& \int_{|x|>R_{0}}(a(x))^{1 / \beta}|u|^{q} \Phi_{\alpha}(u) d x \\
& \leq\|u\|^{n / \beta}\|u\|_{(q-n / \beta) \tau}^{q-n / \beta}\left(\int_{|x|>R_{0}} \Phi_{\alpha}(\gamma u) d x\right)^{1 / \gamma} .
\end{aligned}
$$

Since $(q \beta-n) / \beta \geq n$, by (1.3) and (2.1), we obtain that

$$
\int_{|x|>R_{0}}(a(x))^{1 / \beta}|u|^{q} \Phi_{\alpha}(u) d x<\infty .
$$

From (2.11), (2.12) and (2.15), we have

$$
\int_{\mathbb{R}^{n}} A(x)|u|^{q} \Phi_{\alpha}(u) \mathrm{d} x<\infty .
$$

This completes the first part of the lemma.

Now, when

$$
\alpha\left(\frac{\beta}{\beta-1}\right)^{n /(n-1)}\|u\|^{n /(n-1)}<\alpha_{n},
$$

we can choose $\gamma>\beta^{\prime}=\beta /(\beta-1)$ such that $\alpha \gamma^{n /(n-1)}\|u\|^{n /(n-1)}<\alpha_{n}$ and by (2.2) we get

$$
\int_{|x|>R_{0}} \Phi_{\alpha}(\gamma u) d x \leq C .
$$

Consequently, by (1.3) and (2.14), there exists $C_{1}>0$ such that

$$
\int_{|x|>R_{0}}(a(x))^{1 / \beta}|u|^{q} \Phi_{\alpha}(u) d x \leq C\|u\|^{q} .
$$

From (2.13) and (2.16) we conclude the proof.

\section{The variational framework}

As we mentioned in the introduction, problem (1.1) has variational structure. To apply the critical point theory, we define the functional $I: E \rightarrow \mathbb{R}$ by

$$
I(u)=\frac{1}{n}\|u\|^{n}-\frac{1}{n} \int_{\mathbb{R}^{n}} b(x)|u|^{n} d x-\int_{\mathbb{R}^{n}} g(x) F(u) d x-\varepsilon\langle h, u\rangle .
$$


Using ( $\left.\mathrm{f}_{1}\right)$ and $\left(\mathrm{f}_{2}\right)$, given $\epsilon>0$ for each $\alpha>\alpha_{0}$ and $q \geq n$ there exists $C_{\epsilon}>0$ such that

$$
|f(s)| \leq \epsilon|s|^{n-1}+C_{\epsilon}|s|^{q} \Phi_{\alpha}(s), \quad \text { for all } s \in \mathbb{R} .
$$

Consequently, by the condition $\left(\mathrm{g}_{1}\right)$, we have

$$
\int_{\mathbb{R}^{n}} g(x) F(u) d x \leq \epsilon \Lambda_{0} \int_{\mathbb{R}^{n}} A(x)|u|^{n} d x+C_{\epsilon} \Lambda_{0} \int_{\mathbb{R}^{n}} A(x)|u|^{q+1} \Phi_{\alpha}(u) d x .
$$

Thus, using Lemma 2.2 jointly with Lemma 2.5, it follows that $g(x) F(u) \in$ $L^{1}\left(\mathbb{R}^{n}\right)$ for all $u \in E$. Consequently, $I$ is well defined. Moreover, by Proposition 1 in [16] and standard arguments (see for example [23]), one can see that $I$ is of class $C^{1}$ on $E$. A straightforward calculation shows that, for all $v \in E$,

$$
\begin{aligned}
& \left\langle I^{\prime}(u), v\right\rangle \\
= & \int_{\mathbb{R}^{n}}\left(|\nabla u|^{n-2} \nabla u \nabla v+a(x)|u|^{n-2} u v-b(x)|u|^{n-2} u v-g(x) f(u) v\right) d x-\varepsilon\langle h, v\rangle .
\end{aligned}
$$

Hence, a critical point of $I$ is a weak solution of (1.1) and reciprocally.

The geometric conditions of the mountain-pass theorem for the functional $I$ are established by next lemmas.

Lemma 3.1. Suppose that $\left(\mathrm{a}_{1}\right)-\left(\mathrm{a}_{2}\right),\left(\mathrm{a}_{4}\right),\left(\mathrm{g}_{1}\right),\left(\mathrm{f}_{1}\right)-\left(\mathrm{f}_{2}\right)$ and $\left(\mathrm{b}_{1}\right)$ are satisfied. Then there exists $\varepsilon_{1}>0$ such that for each $0<\varepsilon<\varepsilon_{1}$, there exists $\rho_{\varepsilon}>0$ such that

$$
I(u)>0 \quad \text { if }\|u\|=\rho_{\varepsilon} .
$$

Moreover, $\rho_{\varepsilon}$ can be chosen such that $\rho_{\varepsilon} \rightarrow 0$ as $\varepsilon \rightarrow 0$.

Proof. By using (3.3), Lemma 2.2 and Hölder's inequality, there exist $C_{1}, C_{2}>0$ such that

$$
\begin{aligned}
I(u) \geq & \left(\frac{1}{n}-\epsilon C_{1}\right)\|u\|^{n}-\frac{S_{t_{0}}^{-n}}{n}\|b\|_{\sigma}\|u\|^{n} \\
& -C_{2} \int_{\mathbb{R}^{n}} A(x)|u|^{q+1} \Phi_{\alpha}(u) d x-\varepsilon\|h\|_{E^{\prime}}\|u\| \\
= & \left(\frac{1}{n}-\epsilon C_{1}-\frac{S_{t_{0}}^{-n}}{n}\|b\|_{\sigma}\right)\|u\|^{n} \\
& -C_{2} \int_{\mathbb{R}^{n}} A(x)|u|^{q+1} \Phi_{\alpha}(u) d x-\varepsilon\|h\|_{E^{\prime}}\|u\| .
\end{aligned}
$$

Consequently, if $\|u\|<\rho$ with $\alpha \rho^{n /(n-1)}<\alpha_{n}$, we deduce by Lemma 2.5 that

$$
\begin{aligned}
I(u) & \geq\left(\frac{1}{n}-\epsilon C_{1}-\frac{S_{t_{0}}^{-n}}{n}\|b\|_{\sigma}\right)\|u\|^{n}-C\|u\|^{q+1}-\varepsilon\|h\|_{E^{\prime}}\|u\| \\
& =\left\{\left(\frac{1}{n}-\epsilon C_{1}-\frac{S_{t_{0}}^{-n}}{n}\|b\|_{\sigma}\right)\|u\|^{n-1}-C\|u\|^{q}-\varepsilon\|h\|_{E^{\prime}}\right\}\|u\| .
\end{aligned}
$$


Since $q>n$, we may choose $\varepsilon>0$ is sufficiently small and $\rho>0$ such that

$$
\left(\frac{1}{n}-\epsilon C_{1}-\frac{S_{t_{0}}^{-n}}{n}\|b\|_{\sigma}\right) \rho^{n-1}-C \rho^{q}>0 .
$$

Hence, we can find some $\rho_{\varepsilon}>0$ such that $I(u)>0$ if $\|u\|=\rho_{\varepsilon}$ and even $\rho_{\varepsilon} \rightarrow 0$ as $\varepsilon \rightarrow 0$.

Lemma 3.2. Suppose that $\left(\mathrm{a}_{1}\right)-\left(\mathrm{a}_{2}\right),\left(\mathrm{a}_{4}\right),\left(\mathrm{g}_{1}\right),\left(\mathrm{f}_{1}\right)-\left(\mathrm{f}_{3}\right)$ and $\left(\mathrm{b}_{1}\right)$ are satisfied. Then there exists $e \in E$ with $\|e\|>\rho_{\varepsilon}$ such that

$$
I(e)<\inf _{\|u\|=\rho_{\varepsilon}} I(u) .
$$

Proof. Let $u \in C_{0}^{\infty}\left(\mathbb{R}^{n}\right) \backslash\{0\}, u \geq 0$ with compact support $K=\operatorname{supp}(u)$. By $\left(f_{1}\right)$ and $\left(f_{3}\right)$ there exist $C, d>0$ such that

$$
F(s) \geq C s^{\mu}-d, \quad \text { for all } s \in[0,+\infty) .
$$

By using $\left(\mathrm{g}_{1}\right)$ and $\left(\mathrm{b}_{1}\right)$, we obtain

$$
\begin{aligned}
I(t u) & \leq \frac{t^{n}}{n}\|u\|^{n}-C t^{\mu} \int_{K} g(x) u^{\mu} d x+d \int_{K} g(x) d x-t \varepsilon\langle h, u\rangle \\
& \leq \frac{t^{n}}{n}\|u\|^{n}-C_{1} t^{\mu} \int_{K} u^{\mu} d x+C_{2}(K, g)-t \varepsilon\langle h, u\rangle,
\end{aligned}
$$

for all $t>0$. Since $\mu>n$, we have $I(t u) \rightarrow-\infty$ as $t \rightarrow \infty$. Setting $e=t u$ with $t$ large enough, we get the conclusion.

In order to find an appropriate ball to use minimization argument we need the following result.

Lemma 3.3. Suppose that $\left(\mathrm{a}_{1}\right)-\left(\mathrm{a}_{2}\right),\left(\mathrm{a}_{4}\right),\left(\mathrm{g}_{1}\right),\left(\mathrm{f}_{1}\right)-\left(\mathrm{f}_{2}\right)$ and $\left(\mathrm{b}_{1}\right)$ are satisfied. Then if $h \not \equiv 0$ there exist $\eta>0$ and $v \in E$ with $\|v\|=1$ such that $I(t v)<0$ for all $0<t<\eta$. In particular,

$$
-\infty<c_{0}=\inf _{\|u\| \leq \eta} I(u)<0 .
$$

Proof. Let $v \in E$ be the unique solution of the problem

$$
-\Delta_{n} v+a(x)|v|^{n-2} v=h \quad \text { in } \mathbb{R}^{n} .
$$

Thus, when $h \neq 0$, we have $\langle h, v\rangle=\|v\|^{n}>0$. For $t>0$,

$$
\frac{d}{d t} I(t v)=t^{n-1}\|v\|^{n}-t^{n-1} \int_{\mathbb{R}^{n}} b(x)|v|^{n}-\int_{\mathbb{R}^{n}} g(x) f(t v) v d x-\varepsilon\langle h, v\rangle .
$$

Since $f(0)=0$, by continuity, it follows that there exists $\eta>0$ such that

$$
\frac{d}{d t} I(t v)<0 \quad \text { for all } 0<t<\eta .
$$

Using that $I(0)=0$, it must holds that $I(t v)<0$, for all $0<t<\eta$. 


\section{Minimax level}

In order to get a more precise information about the minimax level obtained by the mountain-pass theorem, we prove the following lemma.

Lemma 4.1. Suppose that rom $\left(a_{1}\right)-\left(a_{3}\right)$ hold. Then $S_{p}$ is attained by a nonnegative function $u_{p} \in E \backslash\{0\}$.

Proof. Let $\left(u_{k}\right)$ be a minimizing sequence of non-negative functions (if necessary, replace $u_{k}$ by $\left.\left|u_{k}\right|\right)$ for $S_{p}$ in $E$, that is,

$$
\int_{\mathbb{R}^{n}}\left|u_{k}\right|^{p} d x=1 \quad \text { and } \quad\left(\int_{\mathbb{R}^{n}}\left(\left|\nabla u_{k}\right|^{n}+a(x)\left|u_{k}\right|^{n}\right) d x\right)^{1 / n} \rightarrow S_{p} .
$$

Then, $\left(u_{k}\right)$ is bounded in $E$ and consequently

$$
\int_{\mathbb{R}^{n}}\left|u_{k}\right|^{p} d x \rightarrow \int_{\mathbb{R}^{n}}\left|u_{p}\right|^{p} d x=1 \quad \text { and } \quad\left\|u_{p}\right\| \leq \liminf _{k \rightarrow+\infty}\left\|u_{k}\right\|=S_{p} .
$$

Thus $S_{p}=\left\|u_{p}\right\|$. This completes the proof of the lemma.

Let us consider the function $\Psi:[0,+\infty) \rightarrow \mathbb{R}$ given by

$$
\Psi(t)=\frac{t^{n}}{n} \int_{\mathbb{R}^{n}}\left(\left|\nabla u_{p}\right|^{n}+a(x)\left|u_{p}\right|^{n}\right) d x-\int_{\mathbb{R}^{n}} g(x) F\left(t u_{p}\right) d x .
$$

Lemma 4.2. Suppose that $\left(\mathrm{g}_{1}\right)$ and $\left(\mathrm{f}_{5}\right)$ hold. Then

$$
\max _{t \geq 0} \Psi(t)<\frac{1}{n}\left(\frac{\beta-1}{n \beta}\right)^{n}\left(\frac{\alpha_{n}}{\alpha_{0}}\right)^{n-1} .
$$

Proof. By Lemma 4.1, it follows that

$$
S_{p}=\left(\int_{\mathbb{R}^{n}}\left(\left|\nabla u_{p}\right|^{n}+a(x)\left|u_{p}\right|^{n}\right) d x\right)^{1 / n} \quad \text { and } \quad \int_{\mathbb{R}^{n}}\left|u_{p}\right|^{p} d x=1 .
$$

On the other hand $\left(\mathrm{g}_{1}\right)$ and $\left(\mathrm{f}_{5}\right)$ imply

$$
\Psi(t) \leq \frac{t^{n}}{n} \int_{\mathbb{R}^{n}}\left(\left|\nabla u_{p}\right|^{n}+a(x)\left|u_{p}\right|^{n}\right) d x-t^{p} \frac{\lambda_{0} C_{p}}{p} \int_{\mathbb{R}^{n}}\left|u_{p}\right|^{p} d x .
$$

By using (4.1) we get

$$
\Psi(t) \leq \frac{t^{n}}{n} S_{p}^{n}-t^{p} \frac{\lambda_{0} C_{p}}{p} \leq \max _{t \geq 0}\left[\frac{t^{n}}{n} S_{p}^{n}-t^{p} \frac{\lambda_{0} C_{p}}{p}\right]=\left(\frac{1}{n}-\frac{1}{p}\right)\left(\frac{S_{p}^{p}}{C_{p} \lambda_{0}}\right)^{n /(p-n)}
$$

and, by $\left(\mathrm{f}_{5}\right)$,

$$
\left(\frac{1}{n}-\frac{1}{p}\right)\left(\frac{S_{p}^{p}}{C_{p} \lambda_{0}}\right)^{n /(p-n)}<\frac{1}{n}\left(\frac{\beta-1}{n \beta}\right)^{n}\left(\frac{\alpha_{n}}{\alpha_{0}}\right)^{n-1} .
$$

Therefore,

$$
\max _{t \geq 0} \Psi(t)<\frac{1}{n}\left(\frac{\beta-1}{n \beta}\right)^{n}\left(\frac{\alpha_{n}}{\alpha_{0}}\right)^{n-1} .
$$


Corollary 4.3. We suppose $\left(a_{1}\right)-\left(a_{4}\right),\left(g_{1}\right),\left(f_{1}\right)-\left(f_{2}\right),\left(f_{5}\right)$ and $\left(b_{1}\right)$. Then

if $\varepsilon>0$ is sufficiently small.

$$
\max _{t \geq 0} I\left(t u_{p}\right)<\frac{1}{n}\left(\frac{\beta-1}{n \beta}\right)^{n}\left(\frac{\alpha_{n}}{\alpha_{0}}\right)^{n-1}
$$

Proof. Since $\left|\varepsilon\left\langle h, u_{p}\right\rangle\right| \leq \varepsilon\|h\|_{E^{\prime}} S_{p}$, taking $\varepsilon$ sufficiently small and using Lemma 4.2, the result follows.

\section{On Palais-Smale sequences}

It is well known that the failure of the (PS) compactness condition creates difficulties in studying this class of elliptic problems involving critical growth and unbounded domains. In next several lemmas we will use and analyze the compactness of Palais-Smale of $I$.

Lemma 5.1. Suppose that $\left(\mathrm{a}_{1}\right),\left(\mathrm{a}_{2}\right),\left(\mathrm{b}_{1}\right)$ and $\left(\mathrm{f}_{3}\right)$ are satisfied. Let $\left(u_{k}\right) \subset E$ be an arbitrary Palais-Smale sequence of I at level c, that is,

$$
I\left(u_{k}\right) \rightarrow c \text { and }\left\|I^{\prime}\left(u_{k}\right)\right\|_{E^{\prime}} \rightarrow 0 .
$$

Then

$$
\left\|u_{k}\right\| \leq C, \quad \int_{\mathbb{R}^{n}} g(x)\left|f\left(u_{k}\right) u_{k}\right| d x \leq C \quad \text { and } \quad \int_{\mathbb{R}^{n}} g(x) F\left(u_{k}\right) d x \leq C .
$$

Proof. Let $\left(u_{k}\right) \subset E$ be a sequence satisfying (5.1), thus for any $\varphi \in E$,

$$
\frac{1}{n}\left\|u_{k}\right\|^{n}-\frac{1}{n} \int_{\mathbb{R}^{n}} b(x)\left|u_{k}\right|^{n} d x-\int_{\mathbb{R}^{n}} g(x) F\left(u_{k}\right) d x-\varepsilon\left\langle h, u_{k}\right\rangle \rightarrow c
$$

and

$$
\begin{aligned}
\mid \int_{\mathbb{R}^{n}}\left[\left|\nabla u_{k}\right|^{n-2} \nabla u_{k} \nabla \varphi\right. & +a(x)\left|u_{k}\right|^{n-2} u_{k} \varphi \\
& \left.-b(x)\left|u_{k}\right|^{n-2} u_{k} \varphi-g(x) f\left(u_{k}\right) \varphi\right]-\varepsilon\langle h, \varphi\rangle \mid \rightarrow 0 .
\end{aligned}
$$

Taking $\varphi=u_{k}$ in (5.3), using $\left(\mathrm{b}_{1}\right)$ and $\left(\mathrm{f}_{3}\right)$ we get

$$
\begin{aligned}
\mu\left(c+\delta_{k}\right)+\varepsilon_{k}\left\|u_{k}\right\|+(\mu-1) \varepsilon\left\langle h, u_{k}\right\rangle \geq\left(\frac{\mu}{n}-1\right)\left\|u_{k}\right\|^{n} & \\
-\int_{\mathbb{R}^{n}} g(x)\left[\mu F\left(u_{k}\right)-f\left(u_{k}\right) u_{k}\right] d x & +\left(1-\frac{\mu}{n}\right) \int_{\mathbb{R}^{n}} b(x)\left|u_{k}\right|^{n} d x \\
& \geq\left(\frac{\mu}{n}-1\right)\left(1-S_{t_{0}}^{-n}\|b\|_{\sigma}\right)\left\|u_{k}\right\|^{n},
\end{aligned}
$$

where $\delta_{k} \rightarrow 0$ and $\varepsilon_{k} \rightarrow 0$. Consequently, $\left\|u_{k}\right\| \leq C$ and from (5.2) and (5.3), we obtain

$$
\int_{\mathbb{R}^{n}} g(x) F\left(u_{k}\right) d x \leq C \quad \text { and } \quad \int_{\mathbb{R}^{n}} g(x)\left|f\left(u_{k}\right) u_{k}\right| d x \leq C .
$$


Thanks to Lemma 2.1 in [8], we have

Lemma 5.2. Let $\Omega \subset \mathbb{R}^{n}$ be a bounded domain and $f: \mathbb{R} \rightarrow \mathbb{R}$ a continuous function. Then, for any sequence $\left(u_{k}\right)$ in $L^{1}(\Omega)$ such that $u_{k} \rightarrow u$ in $L^{1}(\Omega)$,

$$
g(x) f\left(u_{k}\right) \in L^{1}(\Omega) \quad \text { and } \quad \int_{\Omega} g(x)\left|f\left(u_{k}\right) u_{k}\right| d x \leq C_{1},
$$

up to a subsequence, we have

$$
g(x) f\left(u_{k}\right) \rightarrow g(x) f(u) \quad \text { in } \quad L^{1}(\Omega) .
$$

Corollary 5.3. Suppose that $\left(\mathrm{a}_{1}\right)-\left(\mathrm{a}_{4}\right),\left(\mathrm{b}_{1}\right),\left(\mathrm{f}_{1}\right)$ and $\left(\mathrm{f}_{3}\right)$ hold. If $\left(u_{k}\right) \subset E$ is a sequence such that $I\left(u_{k}\right) \rightarrow c$ and $\left\|I^{\prime}\left(u_{k}\right)\right\|_{E^{\prime}} \rightarrow 0$, then $\left(u_{k}\right)$ has a subsequence, still denoted by $\left(u_{k}\right)$ weakly convergent to a weak solution $u \in E$ of problem (1.1). Moreover, when $h \not \equiv 0$ is immediate that $u \not \equiv 0$.

ProOF. Using Lemmas 5.1 and 2.4, up to a subsequence, we have $u_{k} \rightarrow u$ weakly in $E, u_{k} \rightarrow u$ in $L^{s}\left(\mathbb{R}^{n}\right)$ for all $s \in[n,+\infty)$ and $u_{k}(x) \rightarrow u(x)$ almost everywhere in $\mathbb{R}^{n}$. Moreover, arguing as in [12, Lemma 4], we get

$$
\left|\nabla u_{k}\right|^{n-2} \nabla u_{k} \rightarrow|\nabla u|^{n-2} \nabla u \quad \text { weakly in }\left(L^{n /(n-1)}\left(B_{R}\right)\right)^{n}, \quad \text { for all } R>0 .
$$

Therefore, passing to the limit in (5.3) and using Lemma 5.2, we have

$$
\begin{aligned}
\int_{\mathbb{R}^{n}}\left(|\nabla u|^{n-2} \nabla u \nabla \varphi\right. & \left.+a(x)|u|^{n-2} u \varphi\right) d x \\
& -\int_{\mathbb{R}^{n}} b(x)|u|^{n-2} \varphi d x-\int_{\mathbb{R}^{n}} g(x) f(u) \varphi d x-\varepsilon\langle h, \varphi\rangle=0
\end{aligned}
$$

for all $\varphi \in C_{0}^{\infty}\left(\mathbb{R}^{N}\right)$. Since $C_{0}^{\infty}\left(\mathbb{R}^{N}\right)$ is dense in $E$, then $u$ is a weak solution of (1.1). Moreover, when $h \not \equiv 0$, we have immediately that $u \not \equiv 0$.

Lemma 5.4. Suppose that $\left(\mathrm{a}_{1}\right)-\left(\mathrm{a}_{4}\right),\left(\mathrm{b}_{1}\right),\left(\mathrm{f}_{1}\right)-\left(\mathrm{f}_{3}\right)$ are satisfied. If $\left(u_{k}\right)$ is a Palais-Smale sequence for $I$ at any level with

$$
\liminf _{k \rightarrow \infty}\left\|u_{k}\right\|<\frac{\beta-1}{\beta n}\left(\frac{\alpha_{n}}{\alpha_{0}}\right)^{(n-1) / n} .
$$

Then $\left(u_{k}\right)$ possesses a subsequence which converges strongly to a solution $u$ of (1.1).

ProOF. Extracting a subsequence of $\left(u_{k}\right)$ if necessary, we can suppose that

$$
\liminf _{k \rightarrow \infty}\left\|u_{k}\right\|=\lim _{k \rightarrow \infty}\left\|u_{k}\right\|
$$

By Lemma 5.1 and Corollary 5.3 we have that $u_{k} \rightarrow u$ in $E$, where $u$ is a weak solution of (1.1). Writing $u_{k}=u+w_{k}$, it follows that $w_{k} \rightarrow 0$ in $E$. Thus $w_{k} \rightarrow 0$ in $L^{s}\left(\mathbb{R}^{n}\right)$ for $s \in[n,+\infty)$ and $w_{k} \rightarrow 0$ in $L_{\text {loc }}^{s}\left(\mathbb{R}^{n}\right)$ for all $s \geq 1$. Arguing as 
in [12, Lemma 4] we get $\nabla u_{k}(x) \rightarrow \nabla u(x)$ almost everywhere in $\mathbb{R}^{n}$. Thus, by Brezis-Lieb Lemma (see [6]), we obtain

$$
\left\|u_{k}\right\|^{n}=\|u\|^{n}+\left\|w_{k}\right\|^{n}+o_{k}(1) .
$$

We claim that

$$
\int_{\mathbb{R}^{n}} g(x) f\left(u_{k}\right) u d x \rightarrow \int_{\mathbb{R}^{n}} g(x) f(u) u d x .
$$

In fact, since $u \in E$, given $\tau>0$, there exists $\varphi \in C_{0}^{\infty}\left(\mathbb{R}^{n}\right)$ such that $\|\varphi-u\|<\tau$. We can see that

$$
\begin{aligned}
& \left|\int_{\mathbb{R}^{n}} g(x) f\left(u_{k}\right) u d x-\int_{\mathbb{R}^{n}} g(x) f(u) u d x\right| \leq\left|\int_{\mathbb{R}^{n}} g(x) f\left(u_{k}\right)(u-\varphi) d x\right| \\
& \quad+\|\varphi\|_{\infty} \int_{\operatorname{supp} \varphi} g(x)\left|f\left(u_{k}\right)-f(u)\right| d x+\left|\int_{\mathbb{R}^{n}} g(x) f(u)(u-\varphi) d x\right| .
\end{aligned}
$$

Since $\left|\left\langle I^{\prime}\left(u_{k}\right),(u-\varphi)\right\rangle\right| \leq \tau_{k}\|u-\varphi\|$ with $\tau_{k} \rightarrow 0$, we get

$$
\begin{aligned}
& \left|\int_{\mathbb{R}^{n}} g(x) f\left(u_{k}\right)(u-\varphi) d x\right| \\
& \quad \leq \tau_{k}\|u-\varphi\|+\left\|\nabla u_{k}\right\|^{n-1}\|u-\varphi\|+\left(\int_{\mathbb{R}^{n}} a(x)\left|u_{k}\right|^{n} d x\right)^{(n-1) / n}\|u-\varphi\| \\
& \quad+C\|b\|_{\sigma}\left\|u_{k}\right\|^{n-1}\|u-\varphi\|+\varepsilon\|h\|_{E^{\prime}}\|u-\varphi\| \leq C\|u-\varphi\|<C \tau,
\end{aligned}
$$

where $C$ is independent of $k$ and $\tau$. Similarly, using that $\left\langle I^{\prime}(u),(u-\varphi)\right\rangle=0$, we have

$$
\left|\int_{\mathbb{R}^{n}} g(x) f(u)(u-\varphi) d x\right|<C \tau .
$$

By Lemma $5.2, g(x) f\left(u_{k}\right) \rightarrow g(x) f(u)$ in $L_{l o c}^{1}\left(\mathbb{R}^{n}\right)$ and by the previous inequalities, we conclude that

$$
\lim _{k \rightarrow \infty}\left|\int_{\mathbb{R}^{n}} g(x) f\left(u_{k}\right) u d x-\int_{\mathbb{R}^{n}} g(x) f(u) u d x\right|<2 C \tau
$$

and this shows the convergence (5.6) because $\tau$ is arbitrary.

From (5.5) and (5.6), we can write

$$
\left\langle I^{\prime}\left(u_{k}\right), u_{k}\right\rangle=\left\langle I^{\prime}(u), u\right\rangle+\left\|w_{k}\right\|^{n}-\int_{\mathbb{R}^{n}} g(x) f\left(u_{k}\right) w_{k} d x+o_{k}(1),
$$

that is,

$$
\left\|w_{k}\right\|^{n}=\int_{\mathbb{R}^{n}} g(x) f\left(u_{k}\right) w_{k} d x+o_{k}(1) .
$$

From $\left(f_{1}\right)-\left(f_{2}\right)$, given $\epsilon>0$, there exists $C_{\epsilon}>0$ such that

$$
\begin{aligned}
& \left|\int_{\mathbb{R}^{n}} g(x) f\left(u_{k}\right) w_{k} d x\right| \leq \epsilon \Lambda_{0} \int_{\mathbb{R}^{n}} A(x)\left|u_{k}\right|^{n-1}\left|w_{k}\right| d x \\
& \quad+C_{\epsilon} \Lambda_{0} \int_{\mathbb{R}^{n}} A(x)\left|u_{k}\right|^{n} \Phi_{\alpha_{0}+\epsilon}\left(u_{k}\right)\left|w_{k}\right| d x .
\end{aligned}
$$


By Hölder's inequality, Lemma 2.2 and Lemma 2.1 in [27], we have

$$
\begin{aligned}
\int_{\mathbb{R}^{n}} A(x)\left|u_{k}\right|^{n-1}\left|w_{k}\right| d x & \leq\left\|u_{k}\right\|_{L_{A(x)}^{n}\left(\mathbb{R}^{n}\right)}^{n}\left\|w_{k}\right\|_{L_{A(x)}^{n}\left(\mathbb{R}^{n}\right)} \\
& \leq C\left\|u_{k}\right\|^{n-1}\left\|w_{k}\right\|_{L_{A(x)}^{n}\left(\mathbb{R}^{n}\right)}
\end{aligned}
$$

and

$$
\begin{aligned}
& \int_{\mathbb{R}^{n}} A(x)\left|u_{k}\right|^{n} \Phi_{\alpha_{0}+\epsilon}\left(u_{k}\right)\left|w_{k}\right| d x \\
& \quad \leq\left(\int_{\mathbb{R}^{n}} A(x)\left|u_{k}\right|^{n^{2} /(n-1)} d x\right)^{(n-1) / n}\left(\int_{\mathbb{R}^{n}} A(x)\left[\Phi_{\alpha_{0}+\epsilon}\left(u_{k}\right)\right]^{n}\left|w_{k}\right|^{n} d x\right)^{1 / n} \\
& \leq C\left\|u_{k}\right\|^{n}\left(\int_{\mathbb{R}^{n}} A(x) \Phi_{\alpha_{0}+\epsilon}\left(n u_{k}\right)\left|w_{k}\right|^{n} d x\right)^{1 / n} .
\end{aligned}
$$

Since for $\epsilon>0$ sufficiently small we have

$$
\left(\alpha_{0}+\epsilon\right)\left(\frac{n(\beta-\epsilon)}{\beta-\epsilon-1}\right)^{n /(n-1)}\left\|u_{k}\right\|^{n /(n-1)}<\alpha_{n} \quad \text { and } \quad \frac{(\beta n-n)(\beta-\epsilon)}{\epsilon}>n,
$$

arguing as in Lemma 2.5, we obtain for $s$ sufficiently large

$$
\begin{aligned}
\int_{\mathbb{R}^{n}} A(x) \Phi_{\alpha_{0}+\epsilon}\left(n u_{k}\right)\left|w_{k}\right|^{n} d x & \\
& \leq C_{1}\left\|w_{k}\right\|_{n s}^{n}+C_{2}\left\|w_{k}\right\|^{n / \beta}\left(\int_{\mathbb{R}^{n}}\left|w_{k}\right|^{(\beta n-n)(\beta-\epsilon) / \epsilon} d x\right)^{\epsilon / \beta(\beta-\epsilon)} .
\end{aligned}
$$

Therefore, by Lemma (2.4) we get

$$
\int_{\mathbb{R}^{n}} g(x) f\left(u_{k}\right) w_{k} d x \rightarrow 0
$$

Consequently, $\left\|w_{k}\right\| \rightarrow 0$ and the result follows.

Similar to N. Lam and G. Lu [19], we have the following:

Lemma 5.5. Suppose that $\left(\mathrm{a}_{1}\right)-\left(\mathrm{a}_{4}\right)$ and $\left(\mathrm{f}_{1}\right)-\left(\mathrm{f}_{4}\right)$ are satisfied. Let $\left(u_{k}\right) \subset E$ be a Palais-Smale sequence for I with $u_{k} \rightarrow u$ weakly in $E$, then

$$
\int_{\mathbb{R}^{n}} g(x) F\left(u_{k}\right) d x \rightarrow \int_{\mathbb{R}^{n}} g(x) F(u) d x .
$$

Proof. By Lemma 5.2, we have that for all $R>0$,

$$
\int_{B_{R}} g(x) f\left(u_{k}\right) d x \rightarrow \int_{B_{R}} g(x) f(u) d x .
$$

Thus, there exists $l(x) \in L^{1}\left(B_{R}\right)$ such that $g(x) f\left(u_{k}(x)\right) \leq l(x)$ almost everywhere in $B_{R}$.

Let $B=\left\{x \in B_{R}: u_{k}(x) \in\left[0, S_{0}\right]\right.$ for all $\left.k \in \mathbb{N}\right\}$. Then, using $\left(f_{4}\right)$ we can conclude that

$$
g(x) F\left(u_{k}(x)\right) \leq\|g\|_{L^{\infty}\left(B_{R}\right)} \sup _{B} F\left(u_{k}(x)\right)+M_{0} g(x) f\left(u_{k}(x)\right)
$$


almost everywhere in $B_{R}$. By Generalized Lebesgue dominated convergence theorem we obtain

$$
\int_{B_{R}} g(x) F\left(u_{k}\right) d x \rightarrow \int_{B_{R}} g(x) F(u) d x .
$$

In order to prove

$$
\int_{\mathbb{R}^{n}} g(x) F\left(u_{k}\right) \rightarrow \int_{\mathbb{R}^{n}} g(x) F(u) d x
$$

it is sufficient to show that given $\delta>0$, there exists $R>0$ such that

$$
\int_{\mathbb{R}^{n} \backslash B_{R}} g(x) F\left(u_{k}\right) d x<\delta \text { and } \int_{\mathbb{R}^{n} \backslash B_{R}} g(x) F(u) d x<\delta .
$$

To prove it we recall the following facts from our assumptions on the nonlinearity: there exist $C_{1}, C_{2}>0$ such that for $(x, s) \in \mathbb{R}^{n} \times[0,+\infty)$,

$$
\begin{gathered}
g(x) F(s) \leq C_{1} A(x)|s|^{n}+C_{2} g(x) f(s), \\
g(x) F(s) \leq C_{1} A(x)|s|^{n}+C_{2} A(x)|s| \Phi_{\alpha}(s), \\
\int_{\mathbb{R}^{n}} g(x) f\left(u_{k}\right) u_{k} d x \leq C \quad \text { and } \quad \int_{\mathbb{R}^{n}} g(x) F\left(u_{k}\right) d x \leq C .
\end{gathered}
$$

Now, for each $K>0$ we have for any $|s| \leq K$,

$$
\begin{aligned}
g(x) F(s) & \leq C_{1} A(x)|s|^{n}+C_{2} A(x)|s| \Phi_{\alpha}(s) \\
& =C_{1} A(x)|s|^{n}+C_{2} A(x) \sum_{j=n-1}^{+\infty} \frac{\alpha^{j}|s|^{n j /(n-1)+1}}{j !} \\
& \leq C A(x)|s|^{n}\left(1+\sum_{j=n-1}^{+\infty} \frac{\alpha^{j}|K|^{n j /(n-1)+1-n}}{j !}\right) \leq C(\alpha, K) A(x)|s|^{n} .
\end{aligned}
$$

So we get

$$
\begin{aligned}
\int_{\left\{x \in \mathbb{R}^{n} \backslash B_{R}:\left|u_{k}\right| \leq K\right\}} g(x) & F\left(u_{k}\right) d x \leq C(\alpha, K) \int_{\left\{x \in \mathbb{R}^{n} \backslash B_{R}:\left|u_{k}\right| \leq K\right\}} A(x)\left|u_{k}\right|^{n} d x \\
\leq & 2^{n-1} C(\alpha, K) \int_{\left\{x \in \mathbb{R}^{n} \backslash B_{R}:\left|u_{k}\right| \leq K\right\}} A(x)\left|u_{k}-u\right|^{n} d x \\
& +2^{n-1} C(\alpha, K) \int_{\left\{x \in \mathbb{R}^{n} \backslash B_{R}:\left|u_{k}\right| \leq K\right\}} A(x)|u|^{n} d x .
\end{aligned}
$$

Now, using Lemma 2.4 and noticing that $u_{k} \rightarrow u$ weakly in $E$, we can choose $R>0$ such that

$$
\int_{\left\{x \in \mathbb{R}^{n} \backslash B_{R}:\left|u_{k}\right| \leq K\right\}} g(x) F\left(u_{k}\right) d x<\frac{\delta}{3} .
$$


Next, we have

$$
\begin{aligned}
\int_{\left\{x \in \mathbb{R}^{n} \backslash B_{R}:\left|u_{k}\right|>K\right\}} g(x) & F\left(u_{k}\right) d x \leq C_{1} \int_{\mathbb{R}^{n} \backslash B_{R}} A(x)\left|u_{k}\right|^{n} d x \\
& +C_{2} \int_{\left\{x \in \mathbb{R}^{n} \backslash B_{R}:\left|u_{k}\right|>K\right\}} g(x) f\left(u_{k}\right) d x \\
\leq & \frac{C_{1}}{K} \int_{\mathbb{R}^{n} \backslash B_{R}} A(x)\left|u_{k}\right|^{n+1} d x+\frac{C_{2}}{K} \int_{\mathbb{R}^{n}} g(x) f\left(u_{k}\right) u_{k} d x \\
\leq & \frac{C_{1}}{K}\left\|u_{k}\right\|^{n+1}+\frac{C_{2}}{K} \int_{\mathbb{R}^{n}} g(x) f\left(u_{k}\right) u_{k} d x .
\end{aligned}
$$

Thus since $\left\|u_{k}\right\|$ is bounded and by (5.7), we can choose $K$ such that

$$
\int_{\left\{x \in \mathbb{R}^{n} \backslash B_{R}:\left|u_{k}\right|>K\right\}} g(x) F\left(u_{k}\right) d x \leq \frac{C}{K}<\frac{2 \delta}{3} .
$$

Combining all the above estimates, we have

$$
\int_{\mathbb{R}^{n}} g(x) F\left(u_{k}\right) d x \rightarrow \int_{\mathbb{R}^{n}} g(x) F(u) d x,
$$

which completes the proof.

\section{Proof of Theorems 1.1 and 1.2}

First, we will prove the existence of a local minimum type solution.

Proposition 6.1. Under the assumptions $\left(\mathrm{a}_{1}\right)-\left(\mathrm{a}_{4}\right),\left(\mathrm{g}_{1}\right),\left(\mathrm{f}_{1}\right)-\left(\mathrm{f}_{3}\right)$ and $\left(\mathrm{b}_{1}\right)$, there exists $\varepsilon_{1}>0$ such that for each $\varepsilon$ with $0<\varepsilon<\varepsilon_{1}$, equation (1.1) has a minimum type solution $u_{0}$ with $I\left(u_{0}\right)=c_{0}<0$, where $c_{0}$ is defined in (3.4).

Proof. Let $\rho_{\varepsilon}$ be as in Lemma 3.1. We can choose $\varepsilon_{1}>0$ sufficiently small such that

$$
\rho_{\varepsilon}<\frac{\beta-1}{\beta n}\left(\frac{\alpha_{n}}{\alpha_{0}}\right)^{(n-1) / n}, \quad \text { for all } \varepsilon \in\left(0, \varepsilon_{1}\right) .
$$

Since $\bar{B}_{\rho_{\varepsilon}}$ is a complete metric space with the metric given by norm of $E$, convex and the functional $I$ is of class $C^{1}$ and bounded below on $\bar{B}_{\rho_{\varepsilon}}$, by Ekeland's variational principle there is a sequence $\left(u_{k}\right)$ in $\bar{B}_{\rho_{\varepsilon}}$ such that

$$
I\left(u_{k}\right) \rightarrow c_{0}=\inf _{\|u\| \leq \rho_{\varepsilon}} I(u) \text { and }\left\|I^{\prime}\left(u_{k}\right)\right\|_{E^{\prime}} \rightarrow 0 .
$$

Observing that

$$
\left\|u_{k}\right\| \leq \rho_{\varepsilon}<\frac{\beta-1}{\beta n}\left(\frac{\alpha_{n}}{\alpha_{0}}\right)^{(n-1) / n},
$$

by Lemma 5.4 it follows that there is a subsequence of $\left(u_{k}\right)$ which converges strongly to a solution $u_{0}$ of (1.1). Therefore, $I\left(u_{0}\right)=c_{0}<0$.

The proof of the existence of the second solution of (1.1) follows by a standard "mountain-pass" procedure. 
Proposition 6.2. Under the assumptions $\left(\mathrm{a}_{1}\right)-\left(\mathrm{a}_{4}\right),\left(\mathrm{g}_{1}\right),\left(\mathrm{f}_{1}\right)-\left(\mathrm{f}_{5}\right)$ and $\left(\mathrm{b}_{1}\right)$, if $0<\varepsilon<\varepsilon_{1}$ problem (1.1) has a mountain-pass type solution $u_{M}$.

Proof. From Lemma 3.1 and Lemma 3.2, I satisfies the hypothesis of the mountain-pass theorem except possibly the Palais-Smale condition. Thus, using the mountain-pass theorem without the Palais-Smale condition (see [20]), there is a sequence $\left(v_{k}\right)$ in $E$ satisfying

$$
I\left(v_{k}\right) \rightarrow c_{M}>0 \text { and }\left\|I^{\prime}\left(v_{k}\right)\right\|_{E^{\prime}} \rightarrow 0,
$$

where $c_{M}$ is the mountain-pass level. Now, by Corollary 5.3 , the sequence $\left(v_{k}\right)$ converges weakly to a solution $u_{M}$ of (1.1).

Since $v_{k} \rightarrow u_{M}$ weakly in $E$ and $u_{k} \rightarrow u_{0}$ strongly in $E$ we can not conclude that $u_{M}$ and $u_{0}$ are distinct. This will be the goal of the next result.

Proposition 6.3. There exists $\varepsilon_{2}>0$ such that for each $\varepsilon$ with $0<\varepsilon<\varepsilon_{2}$, the solutions of (1.1) obtained in Propositions 6.1 and ?? are distinct.

Proof. By Propositions 6.1 and 6.2, there are sequences $\left(u_{k}\right)$ and $\left(v_{k}\right)$ in $E$ such that

$$
\begin{aligned}
& u_{k} \rightarrow u_{0} \quad \text { in } E, \quad I\left(u_{k}\right) \rightarrow c_{0}<0, \quad\left\|I^{\prime}\left(u_{k}\right)\right\|_{E^{\prime}} \rightarrow 0, \\
& v_{k} \rightarrow u_{M} \quad \text { in } E, \quad I\left(v_{k}\right) \rightarrow c_{M}>0, \quad\left\|I^{\prime}\left(v_{k}\right)\right\|_{E^{\prime}} \rightarrow 0 .
\end{aligned}
$$

Now, suppose by contradiction that $u_{0}=u_{M}$.

Setting

$$
w_{k}=\frac{v_{k}}{\left\|v_{k}\right\|_{1, n}} \quad \text { and } \quad w_{0}=\frac{u_{0}}{\lim _{k \rightarrow \infty}\left\|v_{k}\right\|_{1, n}},
$$

we get $\left\|w_{k}\right\|_{1, n}=1$ and $w_{k} \rightarrow w_{0}$ in $W^{1, n}\left(\mathbb{R}^{n}\right)$. Since the norm is lower semicontinuous with respect to weak convergence, it follows that $\lim _{k \rightarrow \infty}\left\|v_{k}\right\|_{1, n} \geq$ $\left\|u_{0}\right\|_{1, n}>0$. Thus, we have two possibilities:

(i) $\left\|w_{0}\right\|_{1, n}=1$, or

(ii) $\left\|w_{0}\right\|_{1, n}<1$.

If (i) happens, then $\lim _{k \rightarrow \infty}\left\|v_{k}\right\|_{1, n}=\left\|u_{0}\right\|_{1, n}$ and consequently $v_{k} \rightarrow u_{0}$ in $W^{1, n}\left(\mathbb{R}^{n}\right)$. Thus, there exists $\ell \in W^{1, n}\left(\mathbb{R}^{n}\right)$ such that $\left|v_{k}(x)\right| \leq \ell(x)$ almost everywhere in $\mathbb{R}^{n}$ (see [16, Proposition 1]). By (3.2), we have

$$
\left|g(x) f\left(v_{k}\right) v_{k}\right| \leq C_{1} A(x)|\ell|^{n}+C_{2} A(x)|\ell|^{q+1} \Phi_{\alpha}(\ell)
$$

almost everywhere in $\mathbb{R}^{n}$, which is integrable. Then, by Lebesgue dominated convergence theorem we conclude that

$$
\int_{\mathbb{R}^{n}} g(x) f\left(v_{k}\right) v_{k} d x \rightarrow \int_{\mathbb{R}^{n}} g(x) f\left(u_{0}\right) u_{0} d x .
$$


Similarly,

Since

$$
\int_{\mathbb{R}^{n}} g(x) f\left(u_{k}\right) u_{k} d x \rightarrow \int_{\mathbb{R}^{n}} g(x) f\left(u_{0}\right) u_{0} d x
$$

$$
\begin{aligned}
& \left\langle I^{\prime}\left(u_{k}\right), u_{k}\right\rangle=\left\|u_{k}\right\|^{n}-\int_{\mathbb{R}^{n}} g(x) f\left(u_{k}\right) u_{k} d x-\varepsilon\left\langle h, u_{k}\right\rangle \rightarrow 0, \\
& \left\langle I^{\prime}\left(v_{k}\right), v_{k}\right\rangle=\left\|v_{k}\right\|^{n}-\int_{\mathbb{R}^{n}} g(x) f\left(v_{k}\right) v_{k} d x-\varepsilon\left\langle h, v_{k}\right\rangle \rightarrow 0,
\end{aligned}
$$

we conclude that

$$
\lim _{k \rightarrow \infty}\left\|v_{k}\right\|^{n}=\lim _{k \rightarrow \infty}\left\|u_{k}\right\|^{n}=\left\|u_{0}\right\|^{n} .
$$

Therefore, $I\left(v_{k}\right) \rightarrow I\left(u_{0}\right)=c_{0}$ and this is a contradiction with (6.1)-(6.2).

Now, suppose that (ii) happens. Since we can take $\rho_{\varepsilon} \rightarrow 0$ as $\varepsilon \rightarrow 0$, we have that $c_{0} \rightarrow 0$ as $\varepsilon \rightarrow 0$. Thus, there exists $\varepsilon_{2}>0$ such that

$$
\max _{t \geq 0} I\left(t u_{p}\right)<c_{0}+\frac{1}{n}\left(\frac{\beta-1}{n \beta}\right)^{n}\left(\frac{\alpha_{n}}{\alpha_{0}}\right)^{n-1}, \quad \text { for all } \varepsilon \in\left(0, \varepsilon_{2}\right) .
$$

Therefore,

and

$$
\alpha_{0}\left(\frac{n \beta}{\beta-1}\right)^{n /(n-1)}<\frac{\alpha_{n}}{\left[n\left(c_{M}-I\left(u_{0}\right)\right)\right]^{1 /(n-1)}}
$$

(6.3) $\alpha_{0}\left(\frac{n \beta}{\beta-1}\right)^{n /(n-1)}\left\|v_{k}\right\|_{1, n}^{n /(n-1)} \leq \frac{\alpha_{n}}{\left[n\left(c_{M}-I\left(u_{0}\right)\right)\right]^{1 /(n-1)}}\left\|v_{k}\right\|_{1, n}^{n /(n-1)}-\widetilde{\delta}$

for some $\widetilde{\delta}>0$. Since $v_{k} \rightarrow u_{0}$ weakly in $E$, using Lemma 2.4, we obtain

$$
\int_{\mathbb{R}^{n}} b(x)\left|v_{k}\right|^{n} d x \rightarrow \int_{\mathbb{R}^{n}} b(x)\left|u_{0}\right|^{n} d x .
$$

Thus, it follows by Lemma 5.5 that

$$
\begin{aligned}
\frac{1}{n} \lim _{k \rightarrow \infty}\left\|v_{k}\right\|_{1, n}^{n}\left(1-\left\|w_{0}\right\|_{1, n}^{n}\right)=\frac{1}{n} \lim _{k \rightarrow \infty}\left(\left\|v_{k}\right\|_{1, n}^{n}-\left\|u_{0}\right\|_{1, n}^{n}\right) \\
\quad \leq c_{M}-I\left(u_{0}\right)-\frac{1}{n} \int_{\mathbb{R}^{n}} a(x)\left(\left|v_{k}\right|^{n}-\left|u_{0}\right|^{n}\right) d x \leq c_{M}-I\left(u_{0}\right),
\end{aligned}
$$

where we have used the fact that

$$
\int_{\mathbb{R}^{n}} a(x)\left|u_{0}\right|^{n} d x \leq \int_{\mathbb{R}^{n}} a(x)\left|v_{k}\right|^{n} d x .
$$

Thus, for $k$ sufficiently large, we have

$$
\frac{\left\|v_{k}\right\|_{1, n}^{n /(n-1)}}{\left[n\left(c_{M}-I\left(u_{0}\right)\right)\right]^{1 /(n-1)}} \leq \frac{1}{\left(1-\left\|w_{0}\right\|_{1, n}^{n}\right)^{1 /(n-1)}} .
$$

Consequently, by (6.3) we get

$$
\left(\alpha_{0}+\epsilon\right)\left(\frac{n(\beta-\epsilon)}{\beta-\epsilon-1}\right)^{n /(n-1)}\left\|v_{n}\right\|_{1, n}^{n /(n-1)} \leq \frac{\alpha_{n}}{\left(1-\left\|w_{0}\right\|_{1, n}^{n}\right)^{1 /(n-1)}}-\delta
$$


for some $\delta>0$ and $\epsilon>0$ sufficiently small. Now, using (3.2) we get

$$
\begin{aligned}
& \left|\int_{\mathbb{R}^{n}} g(x) f\left(v_{k}\right)\left(v_{k}-u_{0}\right)\right| \\
& \quad \leq \epsilon \int_{\mathbb{R}^{n}} A(x)\left|v_{k}\right|^{n-1}\left|v_{k}-u_{0}\right|+C_{\epsilon} \int_{\mathbb{R}^{n}} A(x)\left|v_{k}\right|^{q} \Phi_{\alpha_{0}+\epsilon}\left(v_{k}\right)\left|v_{k}-u_{0}\right| .
\end{aligned}
$$

By Hölder's inequality and Lemma 2.2, we have

$$
\begin{aligned}
& \int_{\mathbb{R}^{n}} A(x)\left|v_{k}\right|^{n-1}\left|v_{k}-u_{0}\right| d x \leq\left\|v_{k}\right\|_{L_{A(x)}^{n}}^{n-1}\left(\mathbb{R}^{n}\right)\left\|v_{k}-u_{0}\right\|_{L_{A(x)}^{n}\left(\mathbb{R}^{n}\right)} \\
& \leq C\left\|v_{k}\right\|^{n-1}\left\|v_{k}-u_{0}\right\|_{L_{A(x)}^{n}\left(\mathbb{R}^{n}\right)} .
\end{aligned}
$$

Hence, using Lemma 2.4 we get

$$
\int_{\mathbb{R}^{n}} A(x)\left|v_{k}\right|^{n-1}\left|v_{k}-u_{0}\right| d x \rightarrow 0 .
$$

Moreover, using Lemma 2.1 in [27], we have

$$
\begin{aligned}
& \int_{\mathbb{R}^{n}} A(x)\left|v_{k}\right|^{q} \Phi_{\alpha_{0}+\epsilon}\left(v_{k}\right)\left|v_{k}-u_{0}\right| \\
& \leq\left(\int_{\mathbb{R}^{n}} A(x)\left|v_{k}\right|^{q n /(n-1)}\right)^{(n-1) / n}\left(\int_{\mathbb{R}^{n}} A(x)\left[\Phi_{\alpha_{0}+\epsilon}\left(v_{k}\right)\right]^{n}\left|v_{k}-u_{0}\right|^{n}\right)^{1 / n} \\
& \leq C\left\|v_{k}\right\|^{q}\left(\int_{\mathbb{R}^{n}} A(x) \Phi_{\alpha_{0}+\epsilon}\left(n v_{k}\right)\left|v_{k}-u_{0}\right|^{n} d x\right)^{1 / n} .
\end{aligned}
$$

Since

$$
\left(\alpha_{0}+\epsilon\right)\left(\frac{n(\beta-\epsilon)}{\beta-\epsilon-1}\right)^{n /(n-1)}\left\|v_{n}\right\|_{1, n}^{n /(n-1)} \leq \frac{\alpha_{n}}{\left(1-\left\|w_{0}\right\|_{1, n}^{n}\right)^{1 /(n-1)}}-\delta
$$

for some $\delta>0$ and $\epsilon>0$ sufficiently small. Similarly as in the proof of Lemma 2.5 and using Theorem 1.1 in [15], we obtain for $s$ sufficiently large that

$$
\begin{aligned}
\int_{\mathbb{R}^{n}} A(x) \Phi_{\alpha_{0}+\epsilon}\left(n v_{k}\right)\left|v_{k}-u_{0}\right|^{n} d x \leq C_{1}\left\|v_{k}-u_{0}\right\|_{n s}^{n} \\
+C_{2}\left\|v_{k}-u_{0}\right\|^{n / \beta}\left(\int_{\mathbb{R}^{n}}\left|v_{k}-u_{0}\right|^{(\beta n-n)(\beta-\epsilon) / \epsilon} d x\right)^{\epsilon / \beta(\beta-\epsilon)} .
\end{aligned}
$$

For $\epsilon>0$ sufficiently small we have $(\beta n-n)(\beta-\epsilon) / \epsilon>n$. Hence, by Lemma 2.4 and by the previous inequalities, we have

$$
\int_{\mathbb{R}^{n}} g(x) f\left(v_{k}\right)\left(v_{k}-u_{0}\right) d x \rightarrow 0 \quad \text { and } \quad \int_{\mathbb{R}^{n}} b(x)\left|v_{k}\right|^{n-2} v_{k}\left(v_{k}-u_{0}\right) d x \rightarrow 0 .
$$

From these convergence and since $\left\langle I^{\prime}\left(v_{k}\right),\left(v_{k}-u_{0}\right)\right\rangle \rightarrow 0$, we conclude that

$$
\int_{\mathbb{R}^{n}}\left|\nabla v_{k}\right|^{n-2} \nabla v_{k}\left(\nabla v_{k}-\nabla u_{0}\right) d x+\int_{\mathbb{R}^{n}} a(x)\left|v_{k}\right|^{n-2} v_{k}\left(v_{k}-u_{0}\right) d x \rightarrow 0 .
$$


Moreover, since $v_{k} \rightarrow u_{0}$ weakly in $E$, we get

$$
\begin{aligned}
& \int_{\mathbb{R}^{n}}\left|\nabla u_{0}\right|^{n-2} \nabla u_{0}\left(\nabla v_{k}-\nabla u_{0}\right) d x \rightarrow 0, \\
& \int_{\mathbb{R}^{n}} a(x)\left|u_{0}\right|^{n-2} u_{0}\left(v_{k}-u_{0}\right) d x \rightarrow 0 .
\end{aligned}
$$

Using the inequality $\left(|x|^{n-2} x-|y|^{n-2} y\right)(x-y) \geq 2^{2-n}|x-y|^{n}$ for all $x, y \in \mathbb{R}^{n}$, we obtain

$$
\begin{aligned}
\int_{\mathbb{R}^{n}} \mid \nabla v_{k} & -\left.\nabla u_{0}\right|^{n} d x+\int_{\mathbb{R}^{n}} a(x)\left|v_{k}-u_{0}\right|^{n} d x \\
\leq & C_{1} \int_{\mathbb{R}^{n}}\left(\left|\nabla v_{k}\right|^{n-2} \nabla v_{k}-\left|\nabla u_{0}\right|^{n-2} \nabla u_{0}\right)\left(\nabla v_{k}-\nabla u_{0}\right) d x \\
& +C_{2} \int_{\mathbb{R}^{n}} a(x)\left(\left|v_{k}\right|^{n-2} v_{k}-\left|u_{0}\right|^{n-2} u_{0}\right)\left(v_{k}-u_{0}\right) d x
\end{aligned}
$$

From (6.5)-(6.7), we obtain $v_{k} \rightarrow u_{0}$ strongly in $E$. Thus $I\left(v_{k}\right) \rightarrow I\left(u_{0}\right)=c_{0}$, which contradicts (6.1) and (6.2). Therefore $u_{0} \neq u_{M}$.

\section{Proof of Theorem 1.3}

By Lemmas 3.1 and 3.2, I satisfies the hypothesis of the mountain-pass theorem except possibly the Palais-Smale condition. Thus, using the mountainpass theorem without the Palais-Smale condition (see [20]), there is a sequence $\left(v_{k}\right)$ in $E$ satisfying

$$
I\left(v_{k}\right) \rightarrow c_{M} \text { and }\left\|I^{\prime}\left(v_{k}\right)\right\|_{E^{\prime}} \rightarrow 0,
$$

where $c_{M}$ is the mountain-pass level. Now, by Corollary 5.3 , the sequence $\left(v_{k}\right)$ converges weakly to a solution $u_{M}$ of (1.1).

Let us show that $u_{M}$ is nontrivial. Assume, by contradiction, that $u_{M} \equiv 0$. Hence, by Lemma 5.5 we have

$$
\int_{\mathbb{R}^{n}} g(x) F\left(v_{k}\right) d x \rightarrow 0
$$

This together with (5.2) implies that

$$
\left\|v_{k}\right\|^{n} \rightarrow n c_{M}
$$

and hence given $\varepsilon>0$, we have $\left\|v_{k}\right\|^{n} \leq n c_{M}+\varepsilon$, for $k$ sufficiently large. Since

$$
0<c_{M}<\frac{1}{n}\left(\frac{\beta-1}{\beta n}\right)^{n}\left(\frac{\alpha_{n}}{\alpha_{0}}\right)^{n-1}
$$

and choosing $\varepsilon>0$ sufficiently small, we obtain

$$
\left(\alpha_{0}+\varepsilon\right)\left(\frac{\beta-\varepsilon}{\beta-\varepsilon-1}\right)^{n /(n-1)}\left\|v_{k}\right\|^{n /(n-1)}<\alpha_{n}
$$


Consequently, arguing as in Lemma 2.5, we obtain for $s$ sufficiently large that

$$
\begin{aligned}
\int_{\mathbb{R}^{n}} A(x) \Phi_{\alpha_{0}+\varepsilon}\left(v_{k}\right)\left|v_{k}\right|^{n} d x & \\
& \leq C_{1}\left\|v_{k}\right\|_{n s}^{n}+C_{2}\left\|v_{k}\right\|^{n / \beta}\left(\int_{\mathbb{R}^{n}}\left|v_{k}\right|^{(\beta n-n)(\beta-\varepsilon) / \varepsilon} d x\right)^{\varepsilon / \beta(\beta-\varepsilon)} .
\end{aligned}
$$

Since

$\left|\int_{\mathbb{R}^{n}} g(x) f\left(v_{k}\right) v_{k} d x\right| \leq \varepsilon \Lambda_{0} \int_{\mathbb{R}^{n}} A(x)\left|v_{k}\right|^{n} d x+C_{\varepsilon} \Lambda_{0} \int_{\mathbb{R}^{n}} A(x)\left|v_{k}\right|^{n} \Phi_{\alpha_{0}+\varepsilon}\left(v_{k}\right) d x$,

we obtain, by compact embedding $E \hookrightarrow L^{s}\left(\mathbb{R}^{n}\right)$ for $s \in[n,+\infty)$ and by the previous inequalities that

$$
\int_{\mathbb{R}^{n}} g(x) f\left(v_{k}\right) v_{k} d x \rightarrow 0 .
$$

Therefore, by (5.3) with $\varphi=v_{k}$, we achieve $\left\|v_{k}\right\| \rightarrow 0$, which contradicts (7.1) because $c_{M}>0$. Hence, $u_{M}$ is nontrivial and the proof of our result is complete.

\section{REFERENCES}

[1] Adimurthi, Existence of positive solutions of the semilinear Dirichlet problem with critical growth for the N-Laplacian, Ann. Scuola Norm. Sup. Pisa Cl. Sci. 17 (1990), 393-413.

[2] Adimurthi And Y. Yang, An interpolation of Hardy inequality and Trudinger-Moser inequality in $\mathbb{R}^{N}$ and its applications, Internat. Mathematics Research Notices 13 (2010), 2394-2426.

[3] H. Berestycki And P.-L. Lions, Nonlinear scalar field equations I, Arch. Ration. Mech. Anal. 82 (1983), 313-345.

[4] H. Brezis, Functional Analysis, Sobolev Spaces and Partial Differential Equations, Universitext, Springer, New York, 2011.

[5] D.M. CAO, Nontrivial solution of semilinear elliptic equation with critical exponent in $\mathbb{R}^{2}$, Comm. Partial Differential Equations 17 (1992), 407-435.

[6] J. Chabrowski, Variational Methods for Potential Operator Equations. With Applications to Nonlinear Elliptic Equations, de Gruyter Studies in Mathematics, 24. Walter de Gruyter \& Co., Berlin, 1997.

[7] D.G. DE Figueiredo, J.M. DO Ó AND B. RuF, Elliptic equations and systems with critical Trudinger-Moser nonlinearities, Discrete Contin. Dyn. Syst. 30 (2011), 455-476.

[8] D.G. de Figueiredo, O.H. Miyagaki and B. Ruf, Elliptic equations in $\mathbb{R}^{2}$ with nonlinearities in the critical growth range, Calc. Var. Partial Differential Equations 3 (1995), $139-153$

[9] M. DE SouzA, On a singular elliptic problem involving critical growth in $\mathbb{R}^{N}$, NoDEA Nonlinear Differential Equations Appl. 18 (2011), 199-215.

[10] _ Existence and multiplicity of solutions for a singular semilinear elliptic problem in $\mathbb{R}^{2}$, Electron. J. Differential Equations 98 (2011), 1-13.

[11] M. DE Souza And J.M. Do Ó, On a class of singular Trudinger-Moser type inequalities and its applications, Math. Nachr. 284 (2011), 1754-1776.

[12] J.M. Do Ó, Semilinear Dirichlet problems for the $N$-Laplacian in $\mathbb{R}^{N}$ with nonlinearities in critical growth range, Differ. Integral Equ. 9 (1996), 967-979. 
[13] $301-315$

[14] J.M. do Ó, E.S. Medeiros And U.B. Severo, A nonhomogeneous elliptic problem involving critical growth in dimension two, J. Math. Anal. Appl. 345 (2008), 286-304.

[15] J.M. do Ó, M. DE Souza, E. DE Medeiros And U.B. Severo, An improvement for the Trudinger-Moser inequality and applications, J. Differential Equations 256 (2014), 1317-1349.

[16] J.M. do Ó, E. Medeiros And U.B. Severo, On a quasilinear nonhomogeneous elliptic equation with critical growth in $\mathbb{R}^{N}$, J. Differential Equations 246 (2009), 1363-1386.

[17] J. Giacomoni And K. SReenadh, A multiplicity result to a nonhomogeneous elliptic equation in whole space $\mathbb{R}^{2}$, Adv. Math. Sci. Appl. 15 (2005), 467-488.

[18] O. Kavian, Introduction á la Théorie des Points Critiques et Applications aux Problèmes Eelliptiques, Springer-Verlag, Paris, 1993.

[19] N. LAM AND G. LU, Existence and multiplicity of solutions to equations of N-Laplacian type with critical exponential growth in $\mathbb{R}^{N}$, J. Funct. Anal. 262, (2012), 1132-1165.

[20] J. Mawhin and M. Willem, Critical Point Theory and Hamiltonian Systems, SpringerVerlag, Berlin (1989).

[21] J. Moser, A sharp form of an inequality by N. Trudinger, Indiana Univ. Math. J. 20 (1971), 1077-1092.

[22] S.I. Pohozaev, The Sobolev embedding in the case $p l=n$, Proceedings of the Technical Scientific Conference on Advances of Scientific Research 1964-1965, Mathematics Section, Moscov. Energet. Inst. (1965), 158-170.

[23] P.H. Rabinowitz, On a class of nonlinear Schrödinger equations, Z. Angew. Math. Phys. 43 (1992), 270-291.

[24] B. SiRAKOV, Existence and multiplicity of solutions of semi-linear elliptic equations in $\mathbb{R}^{N}$, Calc. Var. Partial Differential Equations 11 (2000), 119-142.

[25] N.S. TRudinger, On the embedding into Orlicz spaces and some applications, J. Math. Mech. 17 (1967), 473-484.

[26] M. Willem, Minimax Theorems, Birkhäuser, Basel, 1996.

[27] Y. YANG, Existence of positive solutions to quasi-linear elliptic equations with exponential growth in the whole Euclidean space, J. Funct. Anal. 262, (2012), 1679-1704.

Manassés de Souza and Jỗo Marcos do Ó

Departamento de Matemática

Universidade Federal da Paraíba

58051-900 João Pessoa, PB, BRAZIL

E-mail address: jmbo@pq.cnpq.br

TARCiAna Silva

Departamento de Matemática

Universidade Federal de Pernambuco

50740-540 Recife, PE, BRAZIL

TMNA : VOLume $45-2015-\mathrm{N}^{\mathrm{O}} 2$ 